\title{
NecroX-5 prevents breast cancer metastasis by AKT inhibition via reducing intracellular calcium levels
}

\author{
JIN-HEE PARK ${ }^{1 *}$, HYOUNG KYU KIM ${ }^{2 *}$, HANA JUNG ${ }^{1}$, KI HYANG KIM ${ }^{3}$, MI SEON KANG ${ }^{4}$, JUN HYUK HONG ${ }^{5}$, \\ BYENG CHUL YU ${ }^{6}$, SUNGJAE PARK ${ }^{3}$, SU-KIL SEO ${ }^{1}$, IL WHAN CHOI ${ }^{1}$, SOON HA KIM ${ }^{6}$, \\ NARI KIM $^{2}$, JIN HAN ${ }^{2}$ and SAE GWANG PARK ${ }^{1}$ \\ Departments of ${ }^{1}$ Microbiology and Immunology, ${ }^{2}$ Physiology, ${ }^{3}$ Internal Medicine and ${ }^{4}$ Pathology, \\ INJE University College of Medicine, Busan 614-735; ${ }^{5}$ Graduate School, Department of Preventive Medicine, \\ Kosin University College of Medicine, Busan 602-702; ${ }^{6}$ LG Life Science, Ltd., \\ R\&D Park, Daejeon 305-380, Republic of Korea
}

Received September 26, 2016; Accepted November 28, 2016

DOI: $10.3892 /$ ijo.2016.3789

\begin{abstract}
A major goal of breast cancer research is to prevent the molecular events that lead to tumour metastasis. It is well-established that both cytoplasmic and mitochondrial reactive oxygen species (ROS) play important roles in cell migration and metastasis. Accordingly, this study examined the molecular mechanisms of the anti-metastatic effects of NecroX-5, a mitochondrial ROS scavenger. NecroX-5 inhibited lung cancer metastasis by ameliorating migration in a mouse model. In human cancer cells, the inhibition of migration by NecroX-5 is cell type-dependent. We observed that the effect of NecroX-5 correlated with a reduction in mitochondrial ROS, but mitochondrial ROS reduction by MitoQ did not inhibit cell migration. NecroX-5 decreased intracellular calcium concentration by blocking $\mathrm{Ca}^{2+}$ influx, which mediated the inhibition of cell migration, AKT downregulation and the reduction of mitochondrial ROS levels. However, the reduction of mitochondrial ROS was not associated with supressed migration and AKT downregulation. Our study demonstrates the potential of NecroX-5 as an inhibitor of breast cancer metastasis.
\end{abstract}

Correspondence to: Dr Sae Gwang Park, Department of Microbiology and Immunology, INJE University College of Medicine, 633-165 GaegumDong, BusanjinGu, Busan 614-735, Republic of Korea E-mail: micpsg@inje.ac.kr

Dr Jin Han, Department of Physiology, INJE University College of Medicine, 633-165 GaegumDong, BusanjinGu, Busan 614-735, Republic of Korea

E-mail: phyhanj@gmail.com

*Contributed equally

Key words: NecroX-5, metastasis inhibition, migration inhibition, mitochondrial ROS, $\mathrm{Ca}^{2+}$

\section{Introduction}

Breast cancer is the most common malignancy and second leading cause of cancer death in women, accounting for $29 \%$ of all female cancers and $15 \%$ of all cancer deaths (1). The main cause of death in breast cancer patients is metastasis (1). Therefore, preventing or controlling the molecular events that lead to tumour metastasis is a major goal in breast cancer research and treatment design. Metastases occur when cancer cells from a primary tumour site spread to distant organs and form new tumours. Metastasis is a multistep process that includes migration and invasion, intravasation, arrest and extravasation, and metastatic colonization (2). Cancer cell migration is induced by various signalling molecules, including transforming growth factor- $\beta$ (TGF- $\beta$ ), integrin, receptor tyrosine kinase, and reactive oxygen species (ROS).

The role of ROS in triggering signalling pathways for cell migration and invasion has been well-established $(3,4)$. ROS, particularly superoxide and peroxide, are constantly generated inside cells by specific enzyme complexes, such as NADPH oxidase (NOX) and nitric oxide synthases (NOS); they are also generated as by-products of oxidation-reduction reactivations, including those underlying mitochondrial respiration (5). In transformed epithelial cells, constitutively activated mitogenic pathways increase intracellular ROS directly or by activating metabolic pathways (6). Additionally, endogenous ROS can be generated by a variety of sources. Mitochondria are major sites of ROS generation in mammalian cells, and mitochondrial ROS (mitoROS) functions as a signalling molecule that stimulates cell proliferation and motility (7). Targeting mitochondria to reduce ROS is an emerging strategy for treating cancer. Goh et al used transgenic mice expressing the mitochondrial catalase and mouse mammary tumour virus-polyoma middle T oncoprotein (MMTV-PyMT) and demonstrated that targeting mitoROS could inhibit tumour progression and prevent metastasis (8).

NecroX-5 is a derivative of the NecroX series of compounds, all of which are mitoROS scavengers (9-11). The chemical composition of NecroX-5 is $\mathrm{C}_{25} \mathrm{H}_{31} \mathrm{~N}_{3} \mathrm{O}_{3} \mathrm{~S} \cdot 2 \mathrm{CH}_{4} \mathrm{O}_{3} \mathrm{~S}$ with a molecular weight of $645.83 \mathrm{~g} / \mathrm{mol}$ (Fig. 1). The therapeutic aim 
of NecroX is to protect cells from necrotic damage caused by CCl4-induced hepatotoxicity, gentamicin-induced ototoxicity, and ischaemic injury in the liver and heart (9-12). However, the anti-tumourigenic effects of NecroX-5 have not been explored to date. In this study, we evaluated whether NecroX-5 can inhibit the metastasis of breast cancer cells and sought to elucidate the molecular mechanisms of this inhibition.

\section{Materials and methods}

Reagents and cell lines.DNase and collagenase (Sigma-Aldrich, St. Louis, MO, USA) were dissolved in Dulbecco's modified Eagle's medium (DMEM). NecroX-5 (LG Life Sciences, Ltd., Seoul, Korea), Mitoquinone (MitoQ; BioTrend, Switzerland), 2',7'-dichlorodihydrofluorescein diacetate $\left(\mathrm{H}_{2} \mathrm{DCFDA}\right)$, MitoSOX-red, Fluo4-AM (Thermo Fisher Scientific, Waltham, MA, USA), MK-2206 (Biovision, Palo Alto, CA, USA), and bepridil (bepridil hydrochloride; Sigma-Aldrich) were dissolved in dimethyl sulphoxide (DMSO) for each condition and dose. Mouse breast cancer 4T1 and human breast cancer HCC70, MDA-MB-231 and MDA-MB-453 cells were purchased from the American Type Culture Collection (ATCC, Manassas, VA, USA). The TUBO-P2J cell line was established from a metastatic lung nodule that had spontaneously developed during a mechanistic study involving an anti-neu antibody in TUBO-bearing mice (13). Cells were grown in DMEM of RPMI with 10\% fetal bovine serum and $1 \%$ penicillin, and cultured at $37^{\circ} \mathrm{C}$ with $5 \% \mathrm{CO}_{2}$.

Animal study and lung colony assay. Six-week-old female BALB/c mice were obtained from Orient Bio (Taejun, Korea). Tumours were established by the subcutaneous injection of $2 \times 10^{5}$ TUBO-P2J cells into the backs of the mice. When the tumour volume reached $100-150 \mathrm{~mm}^{3}$ in the treatment group, NecroX-5 was dissolved in phosphate-buffered saline (PBS) and administered via intraperitoneal injection $(2.5 \mathrm{mg} /$ $\mathrm{kg}$ /day). The same volume of PBS was injected into the control group. Tumour volumes were measured along three orthogonal axes $(\mathrm{x}, \mathrm{y}$ and $\mathrm{z})$ and calculated as tumour volume $=(\mathrm{xyz}) / 2$. All animal procedures were performed in accordance with the animal experimental guidelines set by the Institutional Animal Care and Use Committee of the INJE University College of Medicine (protocol no. 2013-018). Lung metastasis was evaluated using a lung colony assay on day 23 following tumour implantation. Single cell suspensions were prepared from the lung tissue via enzyme digestion using a mixture of DNase $(0.1 \mathrm{mg} / \mathrm{ml})$ and collagenase $(4 \mathrm{mg} / \mathrm{ml})$ and seeded in 48-well culture plates in a 4-fold serial dilution. The plates were incubated in complete DMEM with $500 \mu \mathrm{g} / \mathrm{ml}$ of G418 (Sigma-Aldrich) and stained between days 10 and 14 with crystal violet ( $0.1 \%$ crystal violet acetate).

Migration assay. Breast cancer cell migration was evaluated with 24-well Transwell plates (Falcon, Bedford, MA, USA). The appropriate number of cells was added to the upper chamber and incubated for 6 or $24 \mathrm{~h}$. The upper surface of the membrane was wiped with a cotton-tipped applicator to remove residual cells. Cells in the bottom compartment were fixed and stained with hematoxylin and eosin (H\&E). Cells in four randomly selected fields at $\mathrm{x} 200$ magnification were counted.

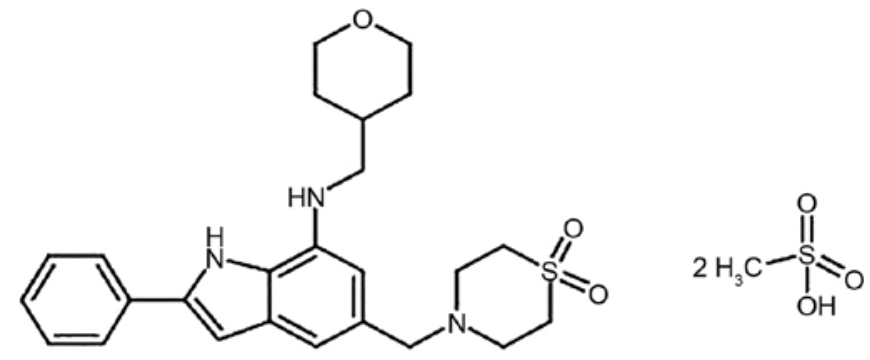

Figure 1. Chemical structure of NecroX-5. NecroX-5 has a chemical composition of $\mathrm{C}_{25} \mathrm{H}_{31} \mathrm{~N}_{3} \mathrm{O}_{3} \mathrm{~S} \cdot 2 \mathrm{CH}_{4} \mathrm{O}_{3} \mathrm{~S}$ with a molecular weight of $645.83 \mathrm{~g} / \mathrm{mol}$.

ROS measurements. Cytoplasmic ROS (cytoROS) was measured with $\mathrm{H}_{2}$ DCFDA. Cells treated with Necrox-5 and MK-2206 for $1 \mathrm{~h}$ were harvested with trypsin $(0.025 \%)$ and stained with $10 \mu \mathrm{M} \mathrm{H}_{2}$ DCFDA for $30 \mathrm{~min}$ at $37^{\circ} \mathrm{C}$. Fluorescence intensity was measured using a FACSCanto II flow cytometer (BD Biosciences, Franklin Lakes, NJ, USA). MitoROS was measured with MitoSOX-red. Cells were treated with NecroX-5, MK-2206 and bepridil for $1 \mathrm{~h}$ in 96-well black microplates (Corning, Inc., Corning, NY, USA) and loaded with $5 \mu \mathrm{M}$ MitoSOX-red. MitoROS levels were detected with a fluorescence reader (Molecular Devices, Sunnyvale, CA, USA) at $510 / 580 \mathrm{~nm}$.

Western blot assay. Whole-cell lysates were prepared in radio-immunoprecipitation assay (RIPA) buffer (Thermo Fisher Scientific) on ice with an added phosphatase inhibitor cocktail (PhosSTOP, cat no. 04906845001; Roche, Basel, Switzerland). The protein concentrations for each sample were determined using the bicinchoninic acid (BCA) protein assay kit (Thermo Fisher Scientific) according to the manufacturer's directions. Equal amounts of protein $(40 \mu \mathrm{g})$ were resolved by electrophoresis on $10 \%$ sodium dodecyl sulphate (SDS) polyacrylamide gels and transferred to nitrocellulose membranes. The membranes were blocked with $5 \%$ non-fat dry milk in TBS and incubated at room temperature for $2 \mathrm{~h}$ with primary antibodies pAKT t308 (monoclonal, rabbit, dilution used 1:1,000, cat no. 2965), pAKT s473 (monoclonal, rabbit, dilution used 1:1,000, cat no. 4058), AKT (polyclonal, rabbit, dilution used 1:2,000, cat no. 9272), PI3K p85 (polyclonal, rabbit, dilution used 1:1,000, cat no. 4292), and pPI3K p110 $\alpha$ (polyclonal, rabbit, dilution used 1:1,000, cat no. 4255) (Cell Signaling Technology). One hour with secondary antibodies were diluted 1:2,000 and incubated for $1 \mathrm{~h}$ (IgG HRP-linked, anti-rabbit and anti-mouse antibodies, cat no. 7074 and 7076, respectively; Cell Signaling Technology). The results were visualized using enhanced chemiluminescent (ECL) detection reagents (Millipore, Darmstadt, Germany).

$\mathrm{Ca}^{2+}$ measurements. The effects of NecroX-5 on intracellular calcium levels were analysed with Fluo-4 AM and a confocal microscope (LSM 700; Carl Zeiss, Oberkochen, Germany). Briefly, harvested cells were washed twice with PBS and incubated at $37^{\circ} \mathrm{C}$ with $5 \mu \mathrm{M}$ Fluo-4 AM (excitation/emission, 494/506 nm). After washing twice with PBS, the stained cells were placed in a perfusion chamber at room temperature. Fluorescence intensity was measured every $30 \mathrm{sec}$ using a confocal microscope with ZEN 2009 software. Regions of 
A

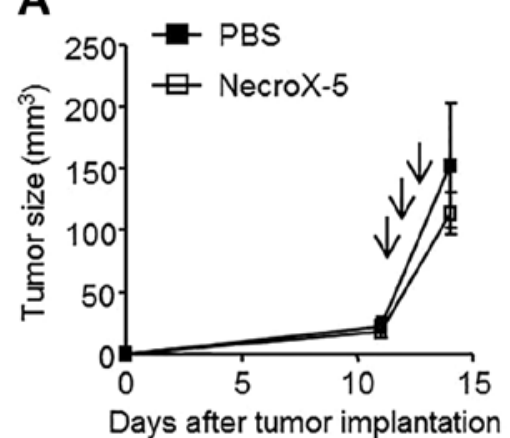

B

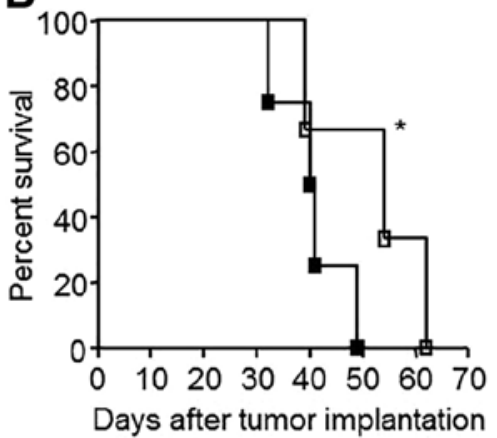

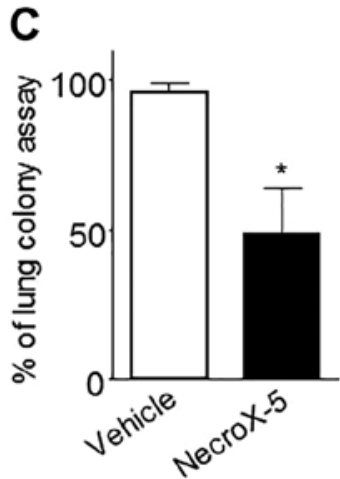

Figure 2. NecroX-5 inhibits in vivo metastasis of breast cancer. TUBO-P2J tumour-bearing mice were treated with NecroX-5 (2.5 mg/kg/day every other day at indicated times). Tumour volumes (A) and survivals of mice (B) were assessed. (C) Lung metastasis was evaluated with the lung colony assay at day 23 following tumour implantation as described in Materials and methods. " $\mathrm{p}<0.05$.
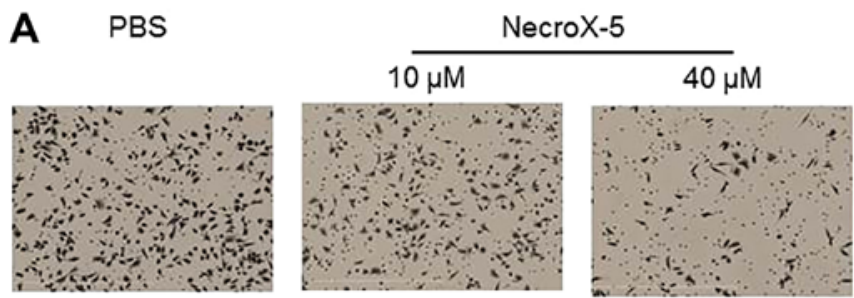

B
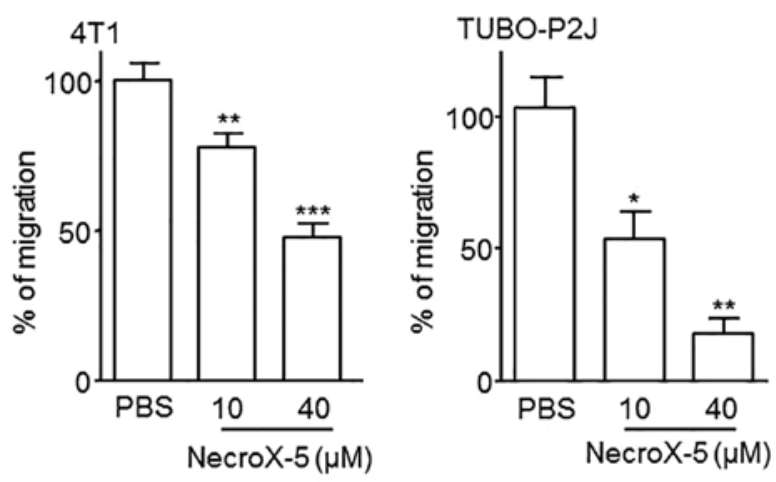

C

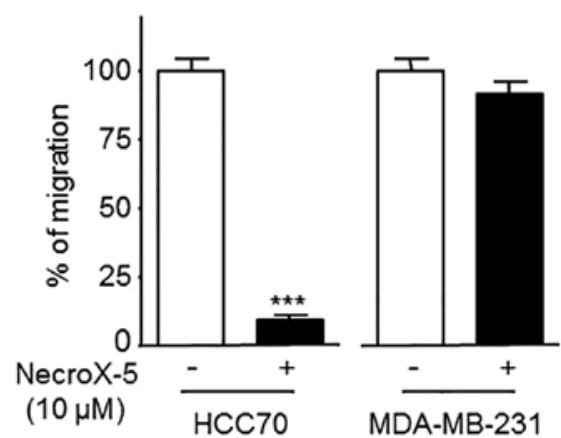

Figure 3. NecroX-5 inhibits tumour cell migration. Breast cancer cells were inoculated in the upper chambers of Transwell plates with $8-\mu \mathrm{m}$ pores with or without NecroX-5 (10 or $40 \mu \mathrm{M})$. After $6 \mathrm{~h}$, the bottom side of membrane was stained with $\mathrm{H} \& \mathrm{E}$ and the cells that had migrated from the upper chamber to the bottom side of the membrane were counted using ten randomly selected fields at x200 magnification. (A) Photograph images of bottom sides of Transwell membrane. (B and C) Statistical analysis of the migration of mouse (B) and human (C) breast cancer cell lines. The results of a representative experiment are presented of more than three independent experiments. ${ }^{*} \mathrm{p}<0.05,{ }^{* *} \mathrm{p}<0.01$ and $^{* * *} \mathrm{p}<0.001$ compared to vehicle or PBS controls.

interest in the cells were selected to monitor changes in fluorescence intensity over time, and background was identified as an area without cells. Fluorescence intensity in cells treated for $10 \mathrm{~min}$ with normal tyrode solution $[143.0 \mathrm{mM} \mathrm{NaCl}, 5.4 \mathrm{mM}$ $\mathrm{KCl}, 1.8 \mathrm{mM} \mathrm{CaCl}_{2}, 0.5 \mathrm{mM} \mathrm{MgCl}, 5.5 \mathrm{mM}$ glucose, and $5.0 \mathrm{mM} \mathrm{N}$-2-hydroxyethylpiperazine-N'-2-ethanesulphonic acid [HEPES, pH 7.4)] was used as the baseline value (F0). Fluorescence intensity during NecroX-5 perfusion $(10 \mu \mathrm{M})$ was recorded every $30 \mathrm{sec}$ for $20 \mathrm{~min}$, and peak values (F1) were analysed relative to the baseline value (F1/F0) after subtracting the autofluorescence in the absence of any fluorescent dye. The temperature was set to $23 \pm 1^{\circ} \mathrm{C}$ while recording. To measure calcium influx, intracellular free $\mathrm{Ca}^{2+}$ was depleted with ethylene glycol tetraacetic acid (EGTA, $3 \mathrm{mM}$ ) and thapsigargin (TG, $2 \mu \mathrm{M}$ ). $\mathrm{Ca}^{2+}$ influx was induced by the subsequent addition of $2 \mathrm{mM} \mathrm{Ca}^{2+}$ with or without NecroX-5 and measured with a fluorescence reader (Molecular Devices) at 485/520 nm.

\section{Results}

NecroX-5 reduces breast cancer cell metastasis by inhibiting cell migration. To test the anticancer effects of NecroX-5, TUBO-P2J tumour-bearing mice were treated three times with $500 \mu \mathrm{g}$ of NecroX-5 every other day. NecroX-5 treatment did not inhibit tumour growth (Fig. 2A), however, it was able to significantly extend the mean survival from 40.5 to 55 days $(\mathrm{p}<0.05)$ (Fig. 2B). The number of cells that metastasized to the lung was also significantly reduced by $60 \%$ (Fig. 2C). These data suggested that NecroX-5 inhibited the metastasis of TUBO-P2J cells, without inhibiting the tumour growth. To determine whether the anti-metastatic effect of NecroX-5 was related to an inhibition of cell migration, we performed a Transwell migration assay. NecroX-5 significantly decreased the migration of the mouse breast cancer cell lines 4T1 and TUBO-P2J, in a dose-dependent manner (Fig. 3A and B). However, NecroX-5 had a different effect on the human breast cancer cells. While it reduced the number of migrated cells in $\mathrm{HCC} 70$, it had no effect in MDA-MB-231 (Fig. 3C).

NecroX-5 indirectly reduces mitoROS levels. To determine whether the observed decrease in cancer cell migration was related to the ROS scavenging activity of NecroX-5, the levels of cytoROS and mitoROS were measured with $\mathrm{H}_{2}$ DCFDA and MitoSOX-red staining, respectively. The intensity of 
A
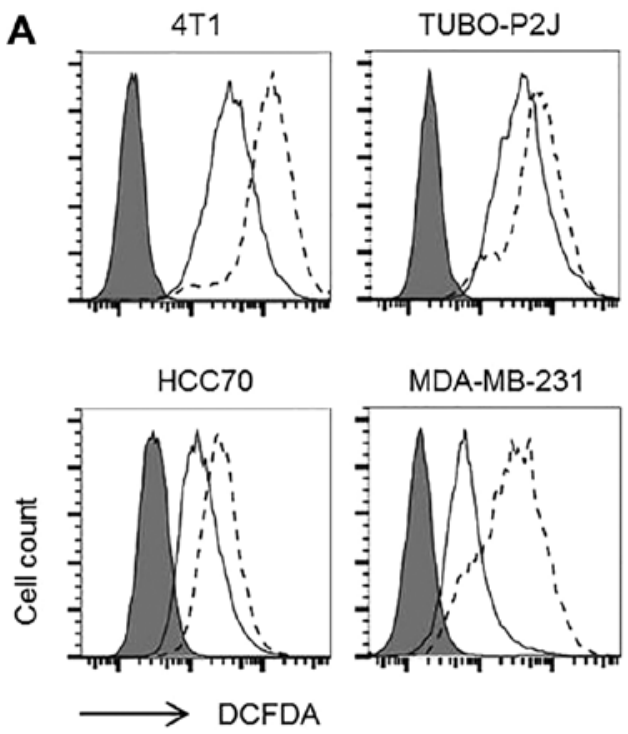

C

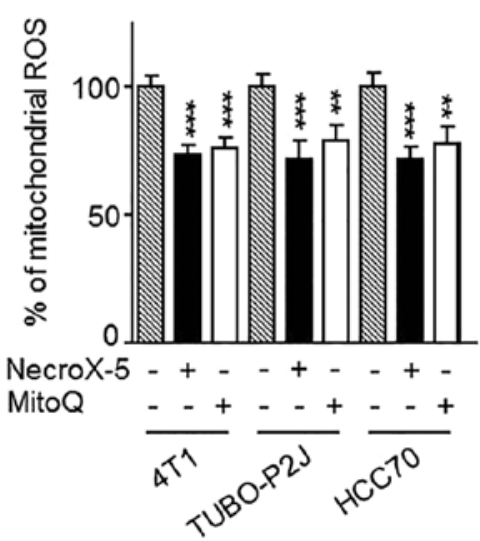

B

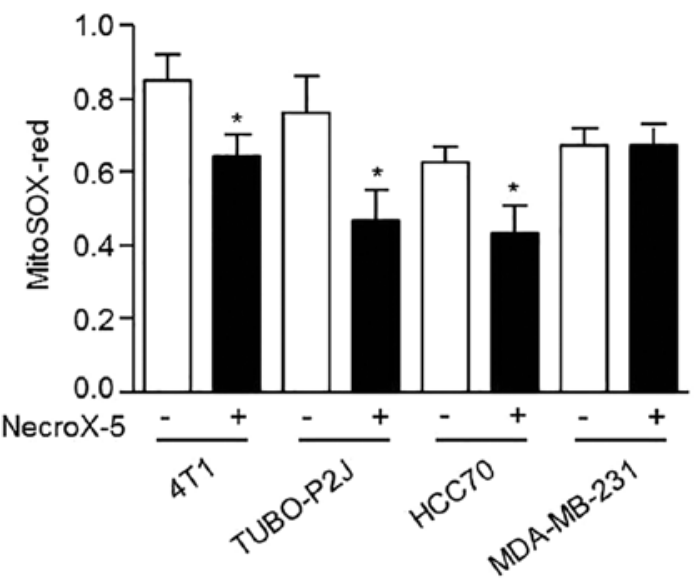

D
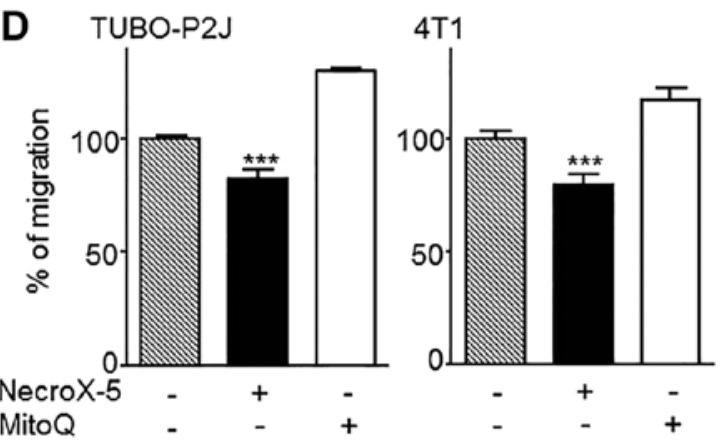

Figure 4. Migration inhibition of NecroX-5 was correlated with mitoROS reduction, but not mediated by that. (A-C) Mouse and human breast cell lines were treated with $10 \mu \mathrm{M}$ of NecroX-5 or $1 \mu \mathrm{M}$ of MitoQ for $1 \mathrm{~h}$ and stained with $\mathrm{H}_{2}$ DCFDA (A) or MitoSOX (B and C). (A) Grey-filled histogram is autofluorescence. Solid line is for NecroX-5 treated cells and dotted line is for PBS treated cells. (D) Migration inhibition effect of MitoQ. The results of a representative experiment are presented of three independent experiments. ${ }^{*} \mathrm{p}<0.05,{ }^{* *} \mathrm{p}<0.01$ and ${ }^{* * *} \mathrm{p}<0.001$ compared to vehicle or PBS controls.

$\mathrm{H}_{2}$ DCFDA decreased following NecroX-5 treatment in all the breast cancer cells tested (Fig. 4A). Interestingly, MDA-MB-231 cells exhibited the most dramatic decrease in $\mathrm{H}_{2}$ DCFDA intensity. Similarly, MitoSOX-red intensities significantly decreased in 4T1, TUBO-P2J and HCC70 cells, but appeared unchanged in the MDA-MB-231 cells, which were not susceptible to NecroX-5 (Fig. 4B). Next, we utilized MitoQ to test whether the observed decrease in migration was modulated by the reduction in mitoROS levels. MitoQ had a similar effect on mitoROS levels as NecroX-5 (Fig. 4C), however, it did not inhibit cell migration (Fig. 4D). These data suggested that the reduction in mitoROS caused by NecroX-5 was not a direct cause of cell migration inhibition.

NecroX-5 reduces intracellular calcium concentrations by limiting calcium influx, which decreases mitoROS levels and inhibited cell migration. NecroX-5 can reduce mitochondrial calcium $\left(\mathrm{Ca}^{2+}\right)$ concentrations, which is important for mitoROS regulation and cell migration $(10,14,15)$. Accordingly, we evaluated changes in intracellular free calcium concentrations using Fluo-4 AM staining. In the HCC70 cells, NecroX-5 decreased intracellular free $\mathrm{Ca}^{2+}$ by $50 \%$ within
20 min (Fig. 5A) but had no effect in MDA-MB-231 (Fig. 5B). To determine whether the decrease in intracellular $\mathrm{Ca}^{2+}$ was mediated by a decrease in $\mathrm{Ca}^{2+}$ influx, 4T1 cells were treated with TG and ethylene glycol tetraacetic acid to chelate $\mathrm{Ca}^{2+}$ and its subsequent influx was followed. NecroX-5-treated cells significantly decreased the $\mathrm{Ca}^{2+}$ influx (Fig. 5C). To test if the decrease in intracellular $\mathrm{Ca}^{2+}$ was related to the biological effect of NecroX-5, we utilized the calcium influx blocker bepridil. Bepridil treatment significantly reduced mitoROS levels (Fig. 5D) and decreased the migratory ability of 4T1, TUBO-P2J and HCC70 cells (Fig. 5E). These data suggest that the effect of NecroX-5 on cancer cell migration and mitoROS levels is mediated by a reduction in intracellular $\mathrm{Ca}^{2+}$ concentrations

NecroX-5 effect on cell migration is mediated by AKT inhibition. One of the differences between the NecroX-5 responsive and non-responsive cell lines was the basal activity of AKT. 4T1, TUBO-P2J and HCC70 responded to NecroX-5 and demonstrated full AKT activation (phosphorylation at Thr308 and Ser473), whereas MDA-MB-231 did not respond to NecroX-5 or display AKT activation (Fig. 6A). 
A

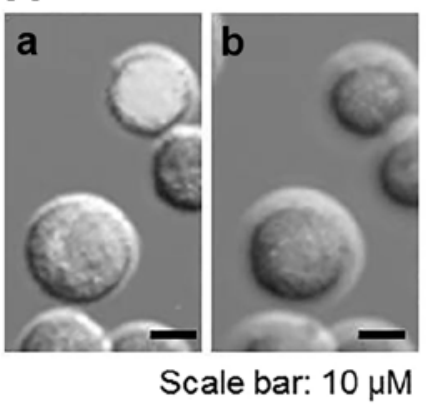

B

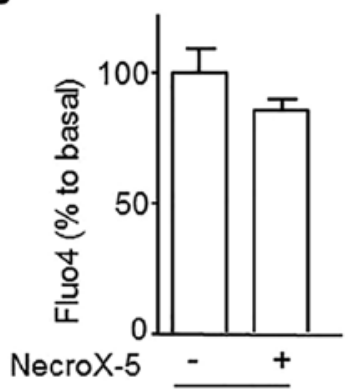

MDA-MB-231
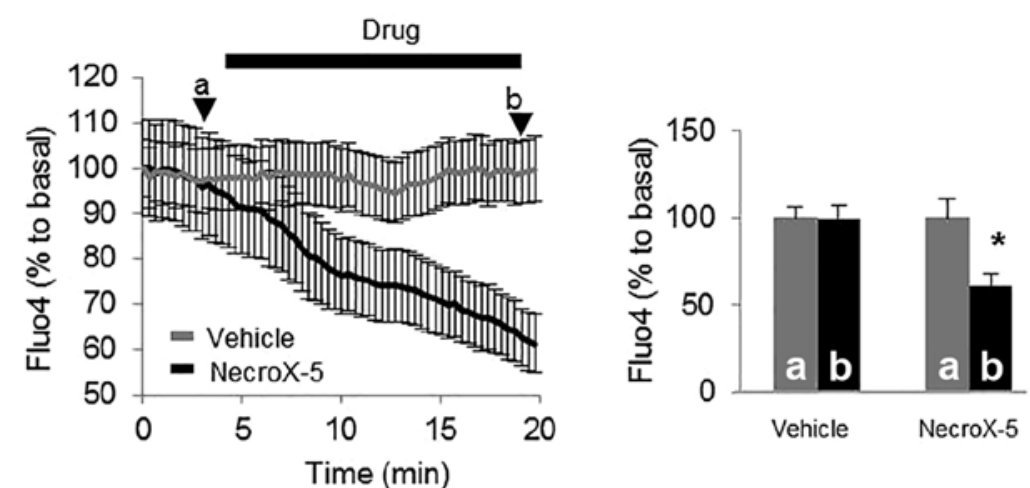

C

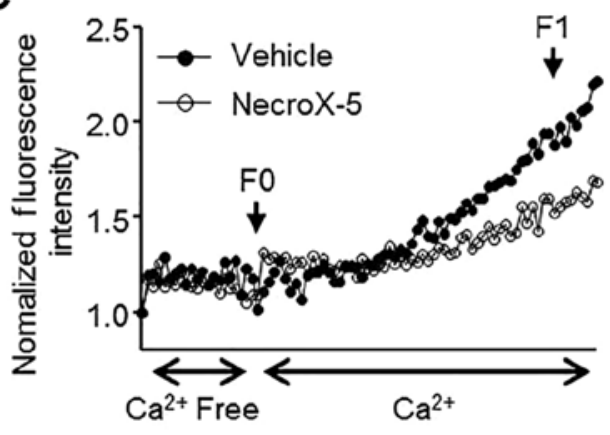

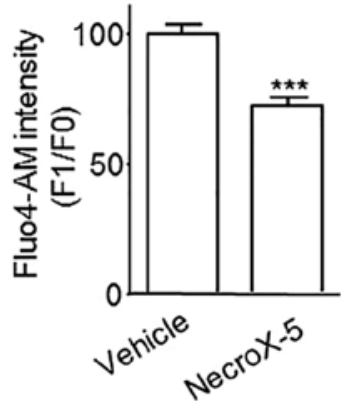

D

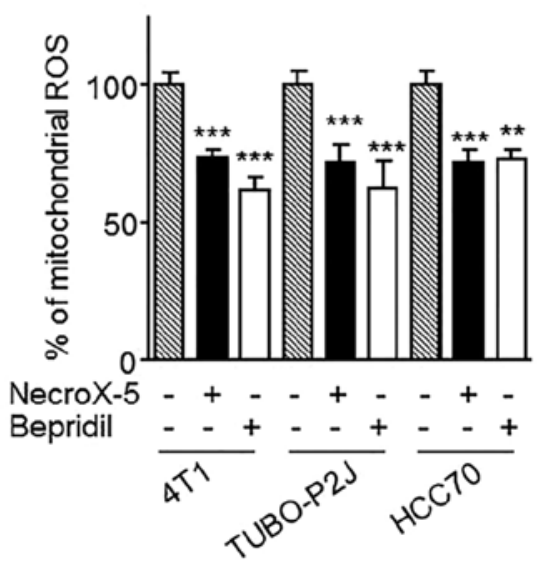

E

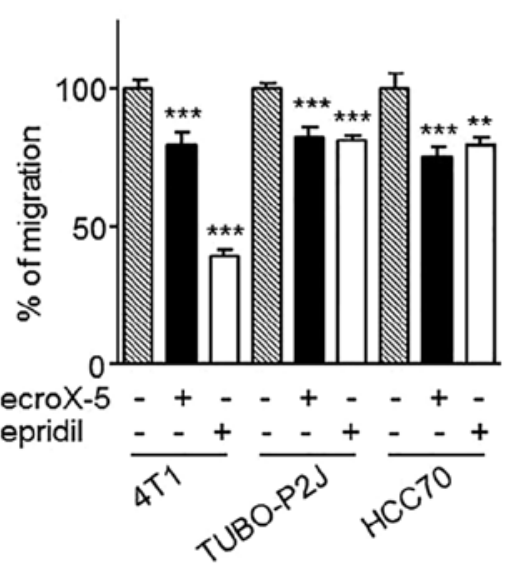

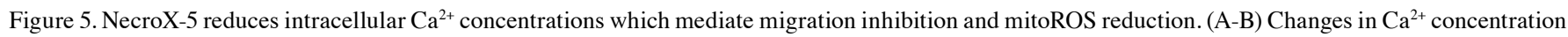
mediated by NecroX-5 in HCC70 (A) and MDA-MD-231 (B) cells. The dynamic changes in intracellular $\mathrm{Ca}^{2+}$ levels measured by the change in Fluo-4AM intensity during the time course and at the indicated times are shown. (C) Changes in $\mathrm{Ca}^{2+}$ influx mediated by NecroX-5 in $4 \mathrm{~T} 1$ cells. Intracellular calcium was depleted with EGTA and TG. The dynamic changes of intracellular $\mathrm{Ca}^{2+}$ levels measured by the change in Fluo-4AM intensity during the time course and at the indicated times are shown. (D) Effects of blocking calcium influx on mitoROS levels. Cells were treated with NecroX-5 (10 $\mu \mathrm{M})$ or bepridil (1 $\mu \mathrm{M})$ for $1 \mathrm{~h}$, and mitoROS levels were measured with MitoSOX-red. (E) Effects of blocking calcium influx on cell migration. Migration was measured with the Transwell migration assay using NecroX-5 $(10 \mu \mathrm{M})$ or bepridil $(1 \mu \mathrm{M}) .{ }^{*} \mathrm{p}<0.05,{ }^{* *} \mathrm{p}<0.01$ and ${ }^{* * *} \mathrm{p}<0.001$ compared to vehicle or PBS controls.

Additionally, treatment with $10 \mu \mathrm{M}$ NecroX-5 significantly reduced the AKT activation levels in all of the responsive cell lines (Fig. 6B). Unlike the downregulation of AKT, pPI3K levels were not changed by NecroX-5 (Fig. 6C). Furthermore, we found this PI3K-independent AKT inhibition was also inducible by bepridil (Fig. 6D). We used an AKT specific inhibitor (MK-2206) to test if AKT inhibition mediated the reduction in cell migration and mitoROS levels. The migratory ability of 4T1 cells was found to be significantly reduced upon treatment with $2.5 \mu \mathrm{M}$ MK-2206 (Fig. 6E). In contrast, MK-2206 did not reduce mitoROS levels in any of the tested cells (Fig. 6F).

\section{Discussion}

This study evaluated the inhibitory effects of NecroX-5 on breast cancer cell metastasis in order to elucidate the NecroX-5 mechanism of action. We demonstrated the inhibitory effects of NecroX-5 on lung metastasis and its ability to extend mouse survival significantly. Additionally, Transwell migration assays revealed that NecroX-5 inhibits cell migration. Interestingly, NecroX-5 did not inhibit migration in any of the breast cancer cell lines tested, leading us to conclude that there were differences in how the cell lines responded to the inhibitory mechanisms of NecroX-5. 
A

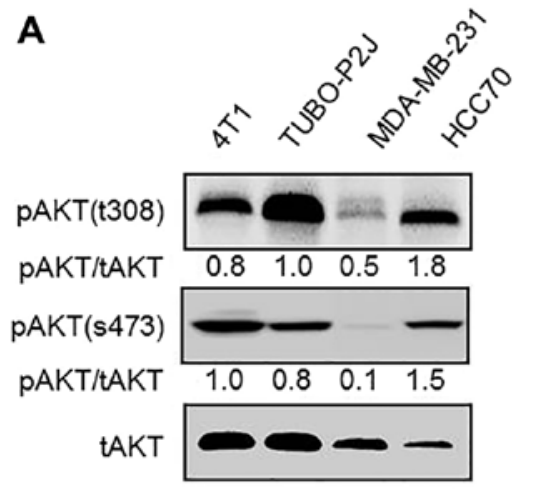

C

TUBO-P2J

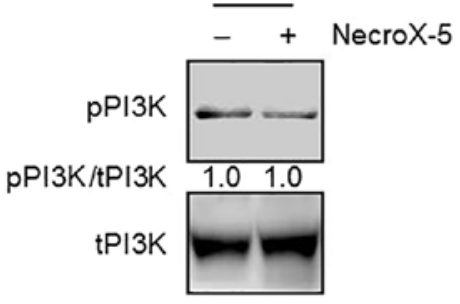

$\mathbf{E}$

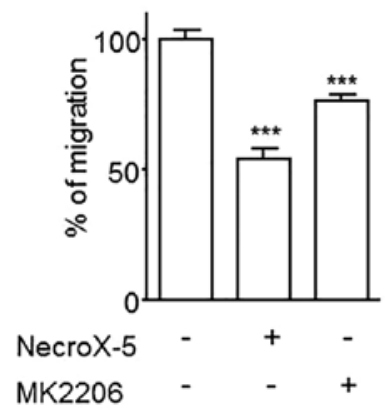

B
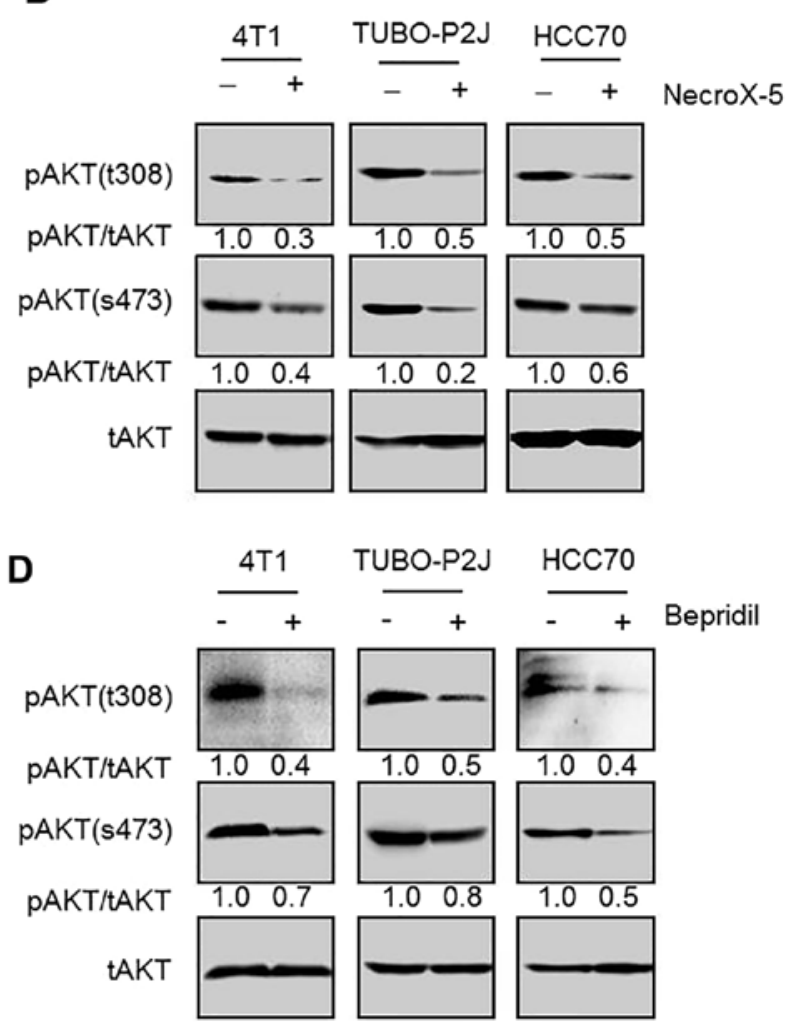

$\mathbf{F}$

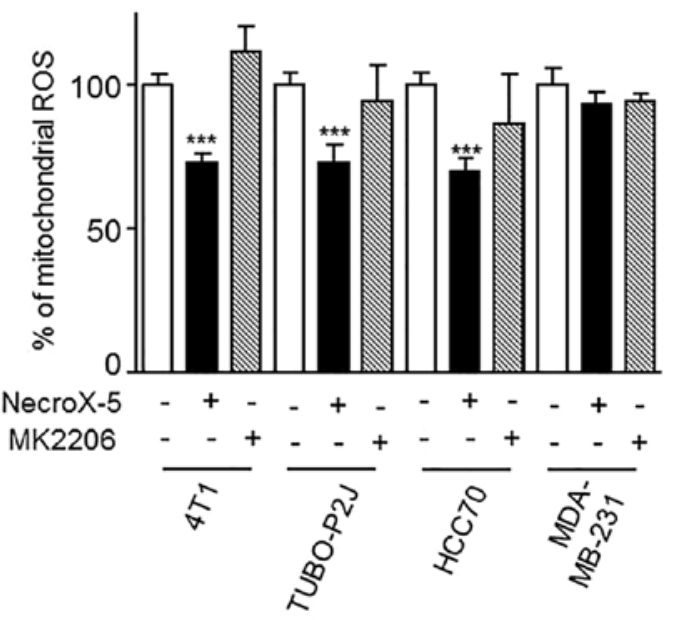

Figure 6. NecroX-5 reduces pAKT levels in a PI3K-independent manner, which mediates the inhibition of cell migration but not a reduction in mitochondrial ROS production. (A-D) Effects of NecroX-5 on PI3K-AKT pathway. Cell lysates were prepared before (A) or after treatment of $10 \mu \mathrm{M}$ of NecroX-5 (B and C) or $1 \mu \mathrm{M}$ of bepridil (D) for $30 \mathrm{~min}$. Western blot analysis was carried out with the indicated antibodies. Ratios of activated/total protein were mean from three independent experiments. (E) Effects of the AKT inhibitor, MK-2206, on cell migration. Migration was measured with Transwell migration assays using either NecroX-5 (10 $\mu \mathrm{M})$ or MK-2206 $(1 \mu \mathrm{M})$. (F) Effects of blocking calcium influx on AKT activation. Cells were treated with $10 \mu \mathrm{M}$ of NecroX-5 or $1 \mu \mathrm{M}$ bepridil for $1 \mathrm{~h}$, and stained with MitoSOX. The results of a representative experiment are presented of two or three independent experiments. ${ }^{* * * *} \mathrm{p}<0.001$ compared to vehicle or PBS controls.

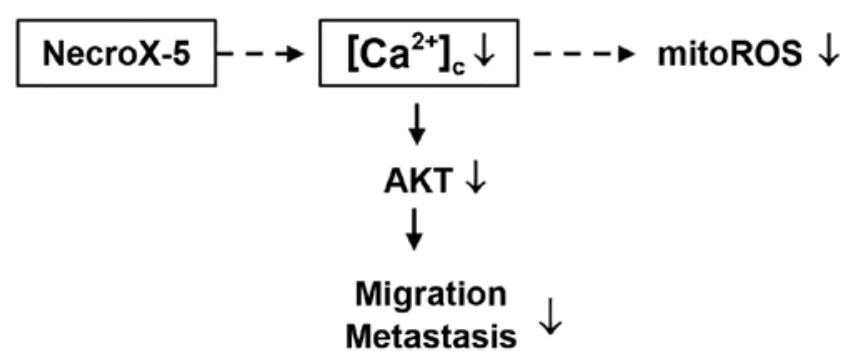

Figure 7. Proposed mechanism of action of NecroX-5.
Cell migration and invasion are the first steps of metastasis. A growing body of evidence suggests that ROS plays important roles in cell migration and invasion (3). Sources of intracellular ROS include the mitochondrial electron transport chain (ETC), phagocytic and non-phagocytic NOX, xanthine oxidases, cyclooxygenases (COX), and lipoxygenases (LOX) (16). However, it is currently unclear which sources are more important for tumour metastasis. In this study, cytoROS and mitoROS were separately evaluated with $\mathrm{H}_{2}$ DCFDA and MitoSOX-red, respectively. NecroX-5 
medicated inhibition of cell migration correlated with the reduced levels of mitoROS, but not cytoROS. This suggested that mitoROS levels are an important target for controlling tumour metastasis. However, other studies have suggested that the inhibition of cell migration and metastasis correlated with reduced cytoROS levels $(8,17-19)$. These differences could be due to differences in experiment settings. Others evaluated migration under specific conditions, whereas we assessed intrinsic migration without any stimulation. Alternatively, NecroX-5 may also inhibit migration by means other than the reduction of mitoROS levels that we identified in this study. Additionally, we concluded that NecroX-5 reduced mitoROS levels indirectly because they remained unchanged in MDA-MB-231 after treatment. Furthermore, experiments with MitoQ demonstrate that reduced mitoROS levels by NecroX-5 may not inhibit cell migration.

MitoROS levels are directly regulated by multiple inputs, including Sirt3, Forkhead box O transcription factors (FOXOs), immunoreceptors, PI3K-AKT, hypoxia, cytokine stimulation, calcium influx, mitophagy and uncoupling proteins (UCPs) (14). Among these inputs, we evaluated changes in the intracellular $\mathrm{Ca}^{2+}$ concentrations because it modulates both ROS generation and clearance $(20,21)$. Moreover, calcium is a critical regulator of cell migration $(15,22-27)$. NecroX-5 has been shown to reduce mitochondrial calcium concentrations (10); our results demonstrate that NecroX-5 significantly reduced intracellular $\mathrm{Ca}^{2+}$ in NecroX-5-responsive cells (HCC70 and 4T1), but not in unresponsive cells (MDA-MB-231). Intracellular $\mathrm{Ca}^{2+}$ levels are determined by a balance between 'on' reactions that introduce $\mathrm{Ca}^{2+}$ into the cytoplasm and 'off' reactions that signal its removal through a coordinated effort of buffers, pumps, and exchangers (28). The 'on' reactions involves calcium influx from the extracellular space and calcium release from intracellular storage in various organelles, including the endoplasmic/sarcoplasmic reticulum (ER/SR), lysosomes, and mitochondria (15). Although we could not rule out other mechanisms that participate in $\mathrm{Ca}^{2+}$ homeostasis, we were able to demonstrate that NecroX-5 reduced $\mathrm{Ca}^{2+}$ influx from the extracellular space. Blocking experiments with bepridil demonstrated that NecroX-5's mechanism of action is to reduce the $\mathrm{Ca}^{2+}$ influx, thereby mediating the reduction of mitoROS levels and the inhibition of cell migration.

We also evaluated the PI3K/AKT pathway because it has been shown to promote cell survival and increase proliferation and motility $(29,30)$. Also NecroX-5 inhibits both cell proliferation and migration in HCC70 cells, and NecroX-5 responsive cell lines exhibited full activation of AKT at baseline (Thr308 and Ser473), while unresponsive cells did not. Western blot analysis revealed that NecroX-5 inhibited AKT in a PI3K-independent and intracellular calcium-dependent manner. In a previous study, AKT was shown to be regulated by calcium/calmodulin in a PI3K-independent manner $(31,32)$. Thus, it is possible that AKT downregulation by NecroX-5 may be mediated by calmodulin inactivation in response to intracellular calcium reduction.

In conclusion, this study introduced NecroX-5 as a novel anticancer drug that inhibits tumour cell metastasis through the $\mathrm{Ca}^{2+}$-dependent modulation of AKT signalling (Fig. 7). Additionally, the downregulation of AKT by blocking calcium influx may be the mechanism by which NecroX-5 inhibits migration. Finally, we found that mitoROS reduction was not related to AKT downregulation (Fig. 7). Future studies should attempt to elucidate the mechanisms by which intracellular calcium concentrations decrease, identify target channels, and evaluate the effects of calcium release in the context of NecroX-5 treatment.

\section{Acknowledgements}

This study was supported by the National Research Foundation of Korea (NRF) and funding granted by the Ministry of Education of Korea (2010-0020224) and by the Ministry of Science, and Future Planning (R13-2007-023-00000-0).

\section{References}

1. Siegel R, Naishadham D and Jemal A: Cancer statistics, 2013. CA Cancer J Clin 63: 11-30, 2013.

2. Steeg PS: Tumor metastasis: Mechanistic insights and clinical challenges. Nat Med 12: 895-904, 2006.

3. Hurd TR, DeGennaro M and Lehmann R: Redox regulation of cell migration and adhesion. Trends Cell Biol 22: 107-115, 2012.

4. Tochhawng L, Deng S, Pervaiz S and Yap CT: Redox regulation of cancer cell migration and invasion. Mitochondrion 13: 246-253, 2013.

5. Pani G, Galeotti T and Chiarugi P: Metastasis: Cancer cell's escape from oxidative stress. Cancer Metastasis Rev 29: 351-378, 2010.

6. Halliwell B: Oxidative stress and cancer: Have we moved forward? Biochem J 401: 1-11, 2007.

7. Storz P: Reactive oxygen species in tumor progression. Front Biosci 10: 1881-1896, 2005.

8. Goh J, Enns L, Fatemie S, Hopkins H, Morton J, Pettan-Brewer C and Ladiges W: Mitochondrial targeted catalase suppresses invasive breast cancer in mice. BMC Cancer 11: 191, 2011.

9. Kim HJ, Koo SY, Ahn BH, Park O, Park DH, Seo DO, Won JH, Yim HJ, Kwak HS, Park HS, et al: NecroX as a novel class of mitochondrial reactive oxygen species and $\mathrm{ONOO}^{-}$scavenger. Arch Pharm Res 33: 1813-1823, 2010.

10. Thu VT, Kim HK, Long T, Lee SR, Hanh TM, Ko TH, Heo HJ, Kim N, Kim SH, Ko KS, et al: NecroX-5 prevents hypoxia/reoxygenation injury by inhibiting the mitochondrial calcium uniporter. Cardiovasc Res 94: 342-350, 2012.

11. Park MK, Lee BD, Chae SW, Chi J, Kwon SK and Song JJ: Protective effect of NecroX, a novel necroptosis inhibitor, on gentamicin-induced ototoxicity. Int J Pediatr Otorhinolaryngol 76: 1265-1269, 2012

12. Choi JM, Park KM, Kim SH, Hwang DW, Chon SH, Lee JH, Lee SY and Lee YJ: Effect of necrosis modulator necrox-7 on hepatic ischemia-reperfusion injury in beagle dogs. Transplant Proc 42: 3414-3421, 2010.

13. Song H, Kim TO, Ma SY, Park JH, Choi JH, Kim JH, Kang MS, Bae SK, Kim KH, Kim TH, et al: Intratumoral heterogeneity impacts the response to anti-neu antibody therapy. BMC Cancer 14: 647, 2014.

14. Sena LA and Chandel NS: Physiological roles of mitochondrial reactive oxygen species. Mol Cell 48: 158-167, 2012.

15. Prevarskaya N, Skryma R and Shuba Y: Calcium in tumour metastasis: New roles for known actors. Nat Rev Cancer 11: 609-618, 2011.

16. Gloire G, Legrand-Poels S and Piette J: NF-kappaB activation by reactive oxygen species: Fifteen years later. Biochem Pharmacol 72: 1493-1505, 2006.

17. Alexandrova AY, Kopnin PB, Vasiliev JM and Kopnin BP: ROS up-regulation mediates Ras-induced changes of cell morphology and motility. Exp Cell Res 312: 2066-2073, 2006.

18. Ishikawa K, Takenaga K, Akimoto M, Koshikawa N, Yamaguchi A, Imanishi H, Nakada K, Honma Y and Hayashi J: ROS-generating mitochondrial DNA mutations can regulate tumor cell metastasis. Science 320: 661-664, 2008 .

19. Nishikawa M: Reactive oxygen species in tumor metastasis Cancer Lett 266: 53-59, 2008.

20. Yan Y, Wei CL, Zhang WR, Cheng HP and Liu J: Cross-talk between calcium and reactive oxygen species signaling. Acta Pharmacol Sin 27: 821-826, 2006. 
21. Zhang DX and Gutterman DD: Mitochondrial reactive oxygen species-mediated signaling in endothelial cells. Am J Physiol Heart Circ Physiol 292: H2023-H2031, 2007.

22. Pettit EJ and Fay FS: Cytosolic free calcium and the cytoskeleton in the control of leukocyte chemotaxis. Physiol Rev 78: 949-967, 1998.

23. Roderick HL and Cook SJ: $\mathrm{Ca}^{2+}$ signalling checkpoints in cancer: Remodelling $\mathrm{Ca}^{2+}$ for cancer cell proliferation and survival. Nat Rev Cancer 8: 361-375, 2008.

24. Clark K, Langeslag M, van Leeuwen B, Ran L, Ryazanov AG, Figdor CG, Moolenaar WH, Jalink K and van Leeuwen FN TRPM7, a novel regulator of actomyosin contractility and cell adhesion. EMBO J 25: 290-301, 2006.

25. Waning J, Vriens J, Owsianik G, Stüwe L, Mally S, Fabian A, Frippiat C, Nilius B and Schwab A: A novel function of capsaicinsensitive TRPV1 channels: Involvement in cell migration. Cell Calcium 42: 17-25, 2007.

26. Hewavitharana T, Deng X, Soboloff J and Gill DL: Role of STIM and Orai proteins in the store-operated calcium signaling pathway. Cell Calcium 42: 173-182, 2007.
27. Yang S, Zhang JJ and Huang XY: Orai1 and STIM1 are critical for breast tumor cell migration and metastasis. Cancer Cell 15: 124-134, 2009

28. Berridge MJ, Bootman MD and Roderick HL: Calcium signalling: Dynamics, homeostasis and remodelling. Nat Rev Mol Cell Biol 4: 517-529, 2003.

29. Cantley LC: The phosphoinositide 3-kinase pathway. Science 296 : $1655-1657,2002$

30. Sullivan LB and Chandel NS: Mitochondrial reactive oxygen species and cancer. Cancer Metab 2: 17, 2014.

31. Coticchia CM, Revankar CM, Deb TB, Dickson RB and Johnson MD: Calmodulin modulates Akt activity in human breast cancer cell lines. Breast Cancer Res Treat 115: 545-560, 2009.

32. Deb TB, Coticchia CM and Dickson RB: Calmodulin-mediated activation of Akt regulates survival of c-Myc-overexpressing mouse mammary carcinoma cells. J Biol Chem 279: 38903-38911, 2004. 